\title{
Script Structures in Modern Audio-Visual Art
}

\author{
Oleksandr Balaban ${ }^{1}$, Olena Levchenko², Ivan Krupskyy ${ }^{3}$, Alla Medvedieva ${ }^{1} \&$ Volodymyr Mykhalov² \\ ${ }^{1}$ Department of Television Journalism and Actor's Mastership, Kyiv National University of Culture and Arts, Kyiv, \\ Ukraine \\ ${ }^{2}$ Department of Cinematography and Television Arts, Kyiv National University of Culture and Arts, Kyiv, Ukraine \\ ${ }^{3}$ Department of Theory and Practice of Journalism, Ivan Franko National University of Lviv, Lviv, Ukraine \\ Correspondence: Oleksandr Balaban, Department of Television Journalism and Actor's Mastership, Kyiv National \\ University of Culture and Arts, Kyiv, 01601, Ukraine. E-mail: balaban5382@toronto-uni.com
}

Received: September 14, 2021

Accepted: October 8, $2021 \quad$ Online Published: October 21, 2021

doi:10.11114/smc.v9i2.5386

URL: https://doi.org/10.11114/smc.v9i2.5386

\begin{abstract}
Every last detail in a work of art of any scale depends on the correctness of its construction. This study reveals the essence of audio-visual text as an artistic area that develops and operates in the modern media landscape. The problem of determining the structure of the audio-visual script at the present stage of development was one of the most significant issues addressed in this study. The purpose of the study is to explore the main stages of creating a script for an audiovisual work as an intricate complex object that is the result of creative and production activities. The primary research method was the method of analysis, which managed to achieve the goal of discussing the current stages of creating and structuring the script of an audio-visual work. The task of compositional construction of the script for an audio-visual work is to unite its elements into one, giving the future work logic, harmony, and integrity. It was established which modern methods of writing a script are the most effective in practice. It was concluded that the use of modern methods of structuring the script in creating an audio-visual product contributes to its improvement as a work of art. The practical significance of the study is that the methodological basis of creating the script presented in this study can be used to construct any audio-visual product.
\end{abstract}

Keywords: sound and visual image, stages of script creation, audio-visual culture, the screen arts, cinema

\section{Introduction}

Scholars are right to believe that forms of the screen arts are gradually replacing traditional ones similar to how audio-visual communication has outshined the printed word. Nowadays, it is difficult to imagine a modern society without an audiovisual culture, which is becoming an integral part of people's lives, a universal communicator, a stimulus for educational activities, and a form of contemporary art. Significant changes influenced by the vibrant and rapid process of technological renewal led to mass computerisation in the field of screen culture. Verbal culture falls back and is replaced by an audiovisual one. Audio-visual arts are an important component of modern media culture as a phenomenon of the information era. In limited interaction with complex and contradictory social processes, the screen has played a crucial role in the democratisation of culture, in the development of its new forms, including new types of audio-visual arts. The screen has become an important factor in education, including higher education, and has contributed to interdisciplinary interaction, humanisation of technical sciences, the development of the new way of thinking, and aesthetic education of the students.

For a long time, the primary aspect of the discourse was the holistic analysis, which appeared in the first half of the 20th century and became the subject matter of scientific reflection itself. Insufficient development of the holistic analysis has led to doubts about its effectiveness and the search for other methods (Abbas, 2019). However, they do not always allow developing a holistic view of the musical text and are often determined by a particular musical-theoretical system. A pedagogically oriented system analysis is proposed as an alternative method that aligns with modern trends in the development of audio-visual culture and is free from described determination. Systematicity is ensured by considering the concept of music as a metasystem. Its procedure, unlike in other methods, has a nonlinear structure. The process of revealing audio-visual content through a system of questions that can be answered through access to systems of harmony, musical form, texture, rhythm, and melody is at its core. The application of this method allows discovering new phenomena and their patterns in the audio-visual text (Zareei, 2020; Martinelli, 2020). 
The synthetic nature of the audio-visual arts plays a special role in the global art culture. Audio-visual arts are a combination of technology and artistic creativity. Technological progress is influencing the language of the audio-visual arts. Its features are massive participation, democratic nature, accessibility, reproduction of the screen arts (cinema, TV, video, multimedia products). The photographic nature of the screen arts is the basis of its material existence. It is no coincidence that foreshortening, general plan, medium, large, light, frame composition, etc. are used in audio-visual arts (Cao, 2020). The synthetic nature of the audio-visual arts is the basis of their figurative specificity. Related arts influence the development of screen language. The potential of the expressiveness of the audio-visual arts depends on the combination of elements of "visible literature", "moving painting", "fine theatre", "light music". The audio-visual arts have gone from black and white and "silent" movies to sound, colour, widescreen, stereo, multimedia, Dolby-Stereo, 3D, etc formats.

Audio-visual (screen) and traditional arts interacted constantly at the turn of the 20th and 21st centuries Akcali, 2020; Binder et al., 2020). Due to new screen technologies (computer art, Internet) a new type of museum has appeared known as "Virtual Museum" and a new version of book culture such as electronic (screen) book. Even theatrical performances, cultural events, and music shows (including philharmonic and opera concerts) are broadcasted via electronic media. It makes audio-visual art in demand within socio-cultural activities (Gegenfurther \& Ebner, 2019). According to popular opinion, the main element of the script is its structure. Aristotle's "Poetics" is the starting point for all the concepts that describe the structure of the script. These theories were developed by authors such as A. Gegenfurtner, C. Ebner (2019), A.C.R. de Amorim [8], R. Elkoshi (2019), H.M. Fayek, A. Kumar (2020), Y. Gambier, H. Jin (2020), Z.-N. Liu, W.-Y. Wang, Y.-P. Yang, Z.-G. Dai (2021). Each of these theories is interesting in its own way. Their only drawback is that almost all of them contradict each other. It is similar to physics, where quantum theory and relativity are good separately but incompatible within the same universe (2019).

\section{Materials and Methods}

The following research methods were chosen to study the script structure of the audio-visual artwork:

1. At the first stage of the study, the method of analysis was used. This method involves the process of imaginary deconstruction of an object into parts to carefully study them according to various criteria. The analysis highlighted the main aspects of the study, described the concept of audio-visual art and the specific features of its creation in modern society, as well as considered the main modern script structures used for creating an audio-visual artwork, formulated the purpose of the study, identified its main stages and structure. The method of analysis allowed achieving the goal of analysing modern structural constructions of the script applied at a modern stage of development of art.

2. In the second stage of the study, a method of synthesis was used It is a combination of parts obtained during the analysis into one entity. Methods of analysis and synthesis are organically interconnected in this study and can take different forms depending on the properties of the subject matter and the purpose of the study. Different types of analysis and synthesis are used depending on the acquired knowledge about the subject matter and understanding of its essence. Direct and empirical analysis and synthesis are used at the stage of the surface level study of the subject matter. In this case, there is the selection of individual parts of the subject matter, the detection of its features, the simplest measurements, fixation of the direct data out of the general information. This type of analysis and synthesis allows investigating the subject matter. Synthesis is widely used as a powerful tool to reach the essence of the studied phenomenon. Here, the operations of analysis and synthesis are not performed mechanically but are based on some theoretical considerations, which can be assumptions about the causal relationship between various phenomena, the action of any pattern. Structural genetic analysis and synthesis allow reaching the essence of the subject matter. This is followed by the assumption about some causal relationships. This type of analysis and synthesis requires identifying such elements in a complex phenomenon that have a decisive influence on all other aspects of the essence of the subject matter.

3. At the third stage, an attempt was made to design a universal model of an audio-visual script, which will help to develop a modern script for any creative project most effectively. For this purpose, the modelling method and the basic principles of designing the script were used. This method allows modelling a specific prototype of a script of modern audio-visual work and determines the impact of each stage on the entire text.

4. The final stage of the subject matter analysis was the evaluation of the effectiveness of the study, where it was necessary to correlate the results and tasks set at the stage of analysis with the data obtained.

\section{Results}

Since ancient times, scholars have attempted to describe the study of established arts within the framework of theoretical knowledge. There were many great thinkers of the past such as G. Hegel, I. Herder, G. Lessing, F. Schelling, as well as modern scientists, among whom S.S. Abbas (2019), Q. Cao (2020), E. Akçalı (2019), R. Elkoshi et al. (2019). It is significant to take into consideration the contemporary scholars' ideas about audio-visual art. The script is the basis for all types and genres of radio and television programmes, feature and documentary films, theatrical productions, and 
theatrical performances. The script (Italian scenario, from Latin scaena - "stage") is a detailed literary description of the action, which is the basis for the creation of the listed creative products. The script is always based on conflict. If there is no conflict, there will be no interest, no personal emotional, and figurative perception of an event (de Amorim, 2018). Working on a script is a complex creative process that requires a screenwriter to perform serious meticulous work. Even after studying the "technology" of creating a script, one cannot be considered a screenwriter, since as with any creative activity this process requires talent, accumulated materials, and continuous work during which the script comes to life and acquires features of the well-rounded work. (Yoshitakam 2018; Nakano et al., 2019; Meikle, 2020).

The script encompasses artistic, psychological, and directing activities. Thus, the screenwriter acts not only as an author of the script and director but also as an artist, psychologist, and organiser. Working on the script should begin with defining the theme and idea. This subject includes a range of phenomena selected and covered by the author. The idea is the main conclusion, opinion, and assessment of the depicted events. The theme is usually set from the beginning, and the idea is conveyed to the audience and participants by the screenwriter and director and represents the main general conclusion. It is necessary to evoke an active perception of the action, to force everyone as though to become the participant of the event, and to comprehend the idea. In this case, the task of understanding the idea is subjected to the development of action. The next step is to consider the composition of the script. Composition is the organisation of the creation of the film, play, programme, as well as the appropriate structured arrangement of the material (Liu et al., 2021).

The artistic structure of the script includes the author's intention, its theme, and idea, composition, image-character. The screenwriter's intention is based on the study of life (Criado, 2019; Blamberger, 2018). Composition is the arrangement, combination, connection, regular construction of the work, the ratio of its individual parts (components) that forming an entity. It is the principle for organising the material. S. Eisenstein wrote that "composition is a construction, which primarily serves to embody the author's attitude to the content and, at the same time, forces the viewer to treat this content in the same way". Composition (from the Latin compositio - "arrangement", "assembly") is immanent for all arts. In "Basics of Screenwriting" S. Gavdis gives the following definition: "Composition organises both the internal construction of the work and its relationship with the environment and the viewer" (Bhui, 2018). Each screenwriter and director give their definition of the composition. However, they are all united by one feature. Composition is the sequential construction of the action in general: from the exposure, through the development of the action and the culmination to the resolution. Thus, the main components of the script are the exposition, the introduction, the main action, the climax, and the finale.

Other elements outside the plot create an atmosphere of action. The detail, the author's remark, and others are of great importance for the script. The artistic structure of the script depends on many components including the personality of the author, the genre of the film, style, era, time of the creation of the work. General requirements for the script are being entertaining, possess flexible expressiveness, being well-edited. The three-act dramaturgical structure is a classical approach, which implies three parts (or acts), in each of which the course of events is consistently developed and the artistic idea of the work is revealed (Ge \& Gao, 2020). For the analysis of the structure of a script, it is necessary to understand what material it is made of. Of course, it's not about the film or the fabric of the screen, but about what the viewer sees on this screen. It is the moving images of certain people and objects that making noises. The lower level of the structure of the film is the same, i.e., the image on the screen, which moves and produces sounds.

Its smallest element is the frame. It is clear that the division of the film into frames is conditional, that there is in-frame editing, that there are whole films shot by one ("Russian Ark") two ("Macbeth"), or several ("Rope") frames. However, in most cases, the frame is the indivisible element, the atom that makes up the substance of the film. A film usually consists of 200 up to 1000 frames. These numbers vary depending on the genre and pace of the film. The next element is the scene. This part of the film is defined by the unity of time, place, and action. Some scholars doubt whether the unity of action matters in this case but these doubts will not be considered in this study (Ghui, 2018). The significant entanglement begins with the transition from scene to episode. For example, there is an episode in the script in which a detective arrests a criminal. It could be solved in one scene. The criminal is sitting at home and enjoying the fruits of his criminal affairs. A detective enters and arrests him. This is the end of the scene. And this is the end of the episode. The genre and typological script structure of audio-visual arts have passed the way of its development. The main types of cinemas that have been developed are documentary, feature, animated (animated), popular science, and educational films. Principles of defining types of cinematography are (Fayek \& Kumar, 2020):

1. The subject presented on the screen, the originality of the cognitive value of the film.

2. Specific features of the fulfilment of the intent, ways of its materialisation into action.

3. The needs of the audience's perception, which is focused on this type of film.

Considering the main events in the production, transmission, presentation, there are several types of compositions:

4. Linear composition is the most common type. It implies that its parts follow one another and adhere to the logic of 
events.

5. Reverse composition is a type, where the events are arranged in reverse logical order even though they follow one another.

6. Circular composition implies that the beginning closes with the finale, i.e., the action begins and ends in the same way.

7. Detective composition means that its exposition is delayed for a long time and there is a constant return to the beginning of events.

8. Montage composition consists of several storylines, which are united by a common semantic problem.

9. Collage composition is one of the most complex types. It is based on a content of a plot that is built on the structural scheme of the whole script, but each part of the script composition (exposition, introduction, culmination, outcome, finale) is revealed by a separate story (Elkoshi, 2019).

Notably, audio-visual creativity has a great variety and exists only as a unity. Different approaches provide for clarification of the possibility of creating a certain composition in this field. In addition, there is a distinction between static (still) and kinematic (related to moving images) forms of creativity. Based on the performed analysis of the typology of media amateurs and the structure of their activities, the idea of the forms of audio-visual creativity in the socio-cultural field was developed. Considering this, the following three types of creative activity are distinguished: primary (spectator, imitation, game); media-productive (camera and editing), and demonstrative (criticism, editing, and review). By analysing the nature of audio-visual creativity, the author highlights the features of the activities which is the basis of this creativity and identifies innovative specifics of forms of vision (direct, instantaneous, virtual, materialised, associative, visualising, integrative, symbolic, predictive, and archetypical), which provide opportunities for creative self-fulfilment, which is very important for both professional and underground video and television studio operators, for working with students of art and culture faculties and colleges.

\section{Discussion}

The structure of the script is the main tool for the screenwriter to tell one's story. All these types of structures are applicable to any genre. According to Y. Gambier (2020), the compositional integrity of the dramatic construction of the script is extremely important. In the script of the cultural and leisure programme, the composition is understood as a combination, a montage of outwardly heterogeneous elements but with deep internal connections. The successful solution of compositional tasks is the most important requirement for revealing the author's ideological and thematic thought. Every script also requires adherence to the basic laws of drama, i.e., it must have a clear compositional construction of the action, which includes such elements as exposition, introduction, development of the action, its culmination, outcome, and finale.

1) the exposition in the script is a special psychological mood of the audience created with the intentionally selected music, thematic exhibitions, advertising, games, an unusual meeting of guests, etc. The exposition in the script usually smoothly grows into the introduction from which the action begins. This may be the first meeting with fairy-tale characters, the first contest of participants, mentioning an obscure fact from the biography of the main guest of the evening or the appearance of a personified image;

2) the main development of the action is the most prominent part of the script since the main plot finds its dramatic progress to the climax here. This part must be subjected to the following laws: each episode of the script must be logically explained, connected by content with the previous and next episodes; the completion of each episode, which must have the logic of construction; the action must be built up to the climax; the series of events must be subordinated to the scriptdirecting course;

3 ) the crucial element of the script composition is the culmination. This is the highest point of developing action at which conflict is resolved. An example of the culmination may be the following moments: raising the flag in honour of the winner; cutting the tape; call for action; ritual part; the most interesting competition, etc. Without culmination, the action flaws monotonously without emotional ups and downs that do not always cause the expected impact on the audience;

4) outcome - the last event, the result of the action, which brings the viewer to understand certain contradictions, and its absence causes a feeling of incompleteness;

5) after the culmination and outcome follows the final part, which possesses a special meaning since it presents the idea in a concentrated form. Therefore, the finale frequently grows into a collective action, which destroys the division between spectators and participants in the audience. The final powerful chord can be a choral performance of songs, chanting, a call, a farewell dance, awarding the winner of the competition, the salute, etc.

The compositional construction of the action in the script has its own laws of subordination of all parts by the principle of harmony. These laws are integrity, contrast, subordination of all means of expression to the ideological plan, proportionality of each episode. Here they are in greater detail: 
1. The law of integrity, interconnection, and subordination of the parts as one. The structural unit of the script is the episode, which reveals a certain feature of the event around which the action unfolds. However, as an independent unit of information, the episode becomes an integral part of the whole. The compositional integrity of the script of the cultural program is achieved by the reasonable number of episodes to reveal the idea, the system of connections between episodes, and content and emotional continuity.

2. The law of contrast is based on the unity and the struggle of opposites which is the basis of dialectics, and the basis of the development, which reveals the contradictions (conflict). Contrast activates the audience's emotional perception of the content of the cultural program, and the constant struggle of positive and negative emotions affects their intellectual and spiritual components.

3. The law of subordination of all means of expression to the ideological thought of the script requires the developed artistic taste, deep knowledge of literature and art, to stay updated, and to possess creative imagination.

4. The law of proportionality. If the previous law describes the qualitative side of the script, the law of proportionality reveals the quantitative ratio of the source material, its distribution by episodes, the significance of the event that is a centre to the cultural program. It is necessary to achieve the proportionality of the location of the main and secondary parts of the script. Their proportionality is both relative to each other and the composition in general. Creative intuition, a sense of proportion, and dramatic logic screenwriter can help the screenwriter to complete this task (Akcali, 2019).

Scriptwriting in modern audio-visual art is a complex, multi-stage, creative process that includes periods of accumulation of informational content, the development of ideas, and writing. The author of the script must combine all its components so that the result is a well-rounded work. The compositional construction of this script has its laws: integrity, interconnection, and subordination of parts as a whole, contrast, subordination of all means of audio-visual work to the script, unity of content and form, proportionality, typification, and generalisation, etc. The task of compositional construction of the script for an audiovisual work is to combine all these elements into one, to give the future work logic, harmony, and integrity. The emotional and aesthetic perception of the audience depends on the extent to which these elements will harmonise with each other, interact in the construction of the plot, follow collateral subordination and complement each other.

\section{Conclusion}

This study investigates not only the specific features of the audio-visual arts (film, television, video, animation, computer graphics, etc.) but also their types, genre structure of the script, and various forms of audio-visual product creation. The purpose of audio-visual creativity is to create an artistic image that includes not only the image-character of the audiovisual work but also the sound-visual image of the work, the image of each episode, scene, and everywhere the visual is transformed into figurative. The most difficult part of the audio-visual creative process is writing a script for film, television, video, which includes several stages: from concept to script to development by the director-producer of the script. The director brings into life one's worldview, life experience, where one embodies the artistic concept of the film, performs the selection of the film crew, which includes cameraman, artist, composer, sound engineer, actors, and all those who create an audio-visual image of the film. Forms of screen creativity include photography. Multimedia forms of creativity in the context of socio-cultural activities are considered carefully. Video technologies within social and cultural activities, as well as computer and multimedia technologies that increase the level of creative activity, are discussed in detail. The audio-visual aspects of screenwriting emphasise the role of vision and thinking, without which audio-visual creativity is impossible. Notably, each stage of audio-visual creativity requires not only knowledge but a clear personal spiritual dominant, associated with natural self-actualisation, the fulfilment of creative potential, which is determined by the content of relevant technologies.

The analysis revealed that the script structure of the audio-visual text is currently emerging in modern society. Hence, the issues related to the development and implementation of the media landscape are promising areas for further research in the academic literature. As a result, the creation of a script for a modern audio-visual work requires knowledge in many creative disciplines such as literature, directing, music. The process of creating a script for an audio-visual work is a complex and time-consuming creative activity.

\section{References}

Abbas, S. S. (2019). Learning dynamic stream weights for coupled-HMM-based audio-visual speech recognition. IEEE/ACM Transactions on Audio Speech and Language Processing, 23(5), 863-876.

Akçalı, E. (2019). Accented essays: documentary as artistic practice in contemporary audiovisual works from Turkey. Critical Arts, 33(2), 42-55. https://doi.org/10.1080/02560046.2019.1671888

Bhui, K. (2018). Passion and reason in the art and science of mental healthcare. British Journal of Psychiatry, 207(1), 8990. https://doi.org/10.1192/bjp.207.1.89-90 
Binder, A., Naderer, B., \& Matthes, J. (2020). Experts, peers, or celebrities? The role of different social endorsers on children's fruit choice. Appetite, 155, article number 104821. https://doi.org/10.1016/j.appet.2020.104821

Blamberger, G. (2018). Imaginations of death and the beyond in India and Europe. Singapore: Springer. https://doi.org/10.1007/978-981-10-6707-5

Cao, Q. (2020). Relationship between the features of Chinese traditional art and aesthetic psychology. Revista Argentina de Clinica Psicologica, 29(1), 797-802.

Criado, J. M. P. (2019). Cuphead and the future of the audio-visual industry. Con A de Animacion, 2019(9), 14-18. https://doi.org/10.4995/caa.2019.11326

De Amorim, A. C. R. (2018). Displacements between curriculum and experimental cinema studies. Praxis Educativa, 13(3), 1025-1043. https://doi.org/10.5212/PraxEduc.v.13i3.0022

Elkoshi, R. (2019). When sounds, colours, and shapes meet: investigating children's audio-visual art in response to classical music. International Journal of Music Education, 37(4), 576-592. https://doi.org/10.1177/0255761419866084

Fayek, H. M., \& Kumar, A. (2020). Large scale audio-visual learning of sounds with weakly labelled data. In: C. Bessiere (Ed.), Proceedings of the Twenty-Ninth International Joint Conference on Artificial Intelligence (pp. 558-565). Redmond: Association for the Advancement of Artificial Intelligence. https://doi.org/10.24963/ijcai.2020/78

Gambier, Y., \& Jin, H. (2020). A connected history of audio-visual translation elements for consideration. Translation Spaces (Netherland), 8(2), 193-230. https://doi.org/10.1075/ts.19011.gam

Ge, W., \& Gao, L. (2020). Research on the training mode of digital art specialty integrating innovation and entrepreneurship education. E3S Web of Conferences, 179, article number 02046. https://doi.org/10.1051/e3sconf/202017902046

Gegenfurtner, A., \& Ebner, C. (2019). Webinars in higher education and professional training: a meta-analysis and systematic review of randomised controlled trials. Educational Research Review, 28, article number 100293. https://doi.org/10.1016/j.edurev.2019.100293

Liu, Z. N., Wang, W. Y., Yang, Y.P., \& Dai, Z. G. (2021). Repeating fast radio bursts from Pulsar-Asteroid Belt collisions. Frequency Drifting and Polarization Astrophysical Journal, 905(2), article number 140. https://doi.org/10.3847/1538-4357/abc55c

Martinelli, D. (2020). What you see is what you hear: creativity and communication in audiovisual texts. New York: Springer International Publishing. https://doi.org/10.1007/978-3-030-32594-7

Meikle, G. (2020). ScreenPlay: a topic-theory-inspired interactive system. Organised Sound, 25(1), 89-105. https://doi.org/10.1017/S1355771819000499

Nakano, Y., Ohshima, H., \& Yamamoto, Y. (2019). Film genre prediction based on film content and screenplay structure. In M. Indrawan-Santiago, E. Pardede, I.L. Salvadori, M. Steinbauer, I. Khalil, G. Anderst-Kotsis (Eds.), ACM International Conference Proceeding Series (pp. 151-155). New York: Association for Computing Machinery. https://doi.org/10.1145/3366030.3366100

Yoshitaka, T. (2018). Audio-visual media and performing arts in danger. In B. Norton, N. Matsumoto (Eds.), Music as Heritage: Historical and Ethnographic Perspectives (pp. 61-78). London: Taylor and Francis. https://doi.org/10.4324/9781315393865-4

Zareei, M. H. (2020). Audio-visual Materialism. Organised Sound, 25(3), 362-371. https://doi.org/10.1017/S1355771820000321

\section{Copyrights}

Copyright for this article is retained by the author(s), with first publication rights granted to the journal.

This is an open-access article distributed under the terms and conditions of the Creative Commons Attribution license which permits unrestricted use, distribution, and reproduction in any medium, provided the original work is properly cited. 